\title{
Mycobacterium bovis tenosynovitis
}

\author{
Jeffrey David Unsworth, ${ }^{1}$ Alec Bonington ${ }^{2}$
}

\begin{abstract}
${ }^{1}$ Acute Pennine NHS Trust, Manchester, UK

${ }^{2}$ Department of Infectous Diseases, North Manchester General Hospital, Manchester, UK
\end{abstract}

\section{Correspondence to}

Dr Jeffrey David Unsworth, jeffreyunsworth7@hotmail.com

\begin{tabular}{|l|}
\hline To cite: Unsworth JD, \\
Bonington A. BMJ Case Rep \\
Published online: [please \\
include Day Month Year] \\
doi:10.1136/bcr-2013- \\
009257 \\
\hline
\end{tabular}

\begin{abstract}
SUMMARY
Infectious tenosynovitis is a rare condition usually presenting with symptoms of joint pain, swelling and deformity. A large number of infectious organisms are known to cause tenosynovitis and prompt and accurate diagnosis is essential to ensure appropriate treatment is delivered before serious complications and functional impairment occurs. We report a case of Mycobacterium bovis tenosynovitis, a rare cause of infectious tenosynovitis; we discuss the clinical features and management of this condition and highlight the difficulties encountered in reaching the correct diagnosis and the importance of the appropriate use of biopsy to aid diagnosis.
\end{abstract}

\section{BACKGROUND}

We present a case of Mycobacterium bovis tenosynovitis, a rare infectious cause of tenosynovitis with only two cases being previously reported. Tenosynovitis refers to inflammation or infection of a tendon and its associated synovial sheath (the synovium), typically presenting with symptoms of pain, swelling and deformity. A host of both infectious and non-infectious conditions may cause tenosynovitis (table 1) and although any joint may be affected, the joints of the hand and wrist are most commonly involved.

While conditions of a non-infectious aetiology such as de Quervain's synovitis and tenosynovitis secondary to repetitive strain are common, infectious causes of tenosynovitis are relatively rare and differentiating between infectious and noninfectious tenosynovitis is often difficult on clinical grounds alone. Further to this, $M$ bovis is not identified on standard culture investigations and does not respond to standard antibiotics; therefore, a low threshold of clinical suspicion is important in diagnosing this rare condition in order to deliver prompt and appropriate treatment before serious complications such as tendon rupture and serious functional impairment occurs.

\section{CASE PRESENTATION}

A 76-year-old woman, a keen gardener with a history of bronchiectasis, presented to her general practitioner with a 4-month history of increasing swelling and pain of the right hand and wrist; initially treated as cellulitis with oral co-amoxiclav but without improvement. Further examination revealed swelling of the right middle finger, especially of the proximal interphalangeal joint, with partial contraction of the palmar fascia. An x-ray was taken, which showed moderate osteoarthritic changes of the distal interphalangeal joint with a lesion of the middle phalanx of the fifth digit, suggestive of an enchondroma. She was referred for an orthopaedic assessment which revealed that she had numbness and pain in the distribution of the median nerve, with Phalen's sign positive, characteristic of carpal tunnel syndrome. She underwent decompressive surgery to alleviate these symptoms and was followed up 2 months later, at which time it was noted that although her symptoms of carpal tunnel syndrome had been cured, the swelling had increased and there was further thickening of the flexor tendon sheath of the middle finger with resulting finger triggering. She underwent surgery to release the trigger finger, during which it was noted that there was florid inflammation involving the flexor tendon sheath, subsequently confirmed to be a granulomatous synovitis upon histological analysis, suggested to be sarcoidosis by the histologists (staining for Acid and Alcohol Fast Bacilli (AAFBs) was negative, but unfortunately culture for tuberculosis (TB) was not undertaken at this stage).

She was referred for a rheumatological opinion (now 10 months post onset of her symptoms), who suggested that although the localised slowly progressive tenosynovitis found in this lady was not typical of sarcoidosis, further investigation was warranted. Serum ACE was within normal limits, chest $\mathrm{x}$-ray showed no hilar lymphadenopathy and a high-resolution CT scan revealed bronchiectic changes and the identification of an enlarged axillary lymph node ipsilateral to the affected hand measuring approximately $2 \mathrm{~cm}$ in size. Input from the surgical team was requested for them to undertake a biopsy in order to send for histology and TB culture. However, the lymph node was too small for this to be successfully undertaken.

\section{DIFFERENTIAL DIAGNOSIS}

She was referred for assessment by the infectious disease team (now 12 months post onset of her symptoms). At this time her symptoms had progressed and there was found to be a significant amount of swelling over her right wrist joint extending $8-10 \mathrm{~cm}$ proximally (figures 1 and 2). There was also swelling over the palmar aspect of the hand and she was unable to extend her fingers. She had remained afebrile over the preceding 12 months and denied any history of night sweats, fever or respiratory symptoms; however, she did state that she had lost approximately 1 stone in weight over the preceding 12 months despite a good appetite. There was no history of the keeping of tropical fish (Mycobacterium marinum), she owned no pets nor had any history of dog or cat bites/scratches (Capnocytophaga canimorsus, Bartonella henselae, Staphylococcus aureus and Pasteurella multocida) and there was no recent history of foreign travel, having only visited Japan, 
Table 1 Infectious and non-infectious causes of tenosynovitis

\begin{tabular}{ll}
\hline Non-infectious causes & Infectious causes \\
\hline Amyloidosis & Staphylococcus species \\
Crystalline deposition & Streptococcus species \\
De Quervain's tenosynovitis & Eikenella corrodens \\
Diabetes mellitus & Pasteurella multocida \\
Ochronosis & Haemophilus species \\
Overuse syndromes & Capnocytophaga canimorsus \\
Psoriatic arthritis & Mycobacterium species \\
Rheumatoid arthritis & Neisseria gonorrhoeae \\
Sarcoidosis & Pseudomonas aeruginosa \\
Systemic lupus erythematosus & Listeria monocytogenes \\
\hline The list is not exhaustive. Non-infectious causes are listed alphabetically.
\end{tabular}

Singapore and Australia, over 10 years previous. She did, however, live very close to a farm as a child and was closely involved in various farming activities including the milking of cows and she had frequently consumed unpasteurised milk during her childhood. Blood analyses for full blood count, urea and electrolytes and liver function tests were essentially unremarkable except for an isolated raised corrected calcium ( $2.66 \mathrm{mmol} / \mathrm{L}$; although she was receiving calcium supplementation at the time). C reactive protein was persistently within the normal range and serological tests for Syphilis, Brucellosis, Lyme disease, Bartonella spp and Coxiella burnetii were all negative.

The differential diagnosis of the granulomatous tenosynovitis at this time was either that of sarcoidosis or a mycobacterial infection, which posed some management difficulties because empirical immunosuppression therapy for sarcoidosis in the presence of a mycobacterial infection would be detrimental. Further information was required; she underwent exploration and washout biopsy due to the development of a pointed abscess on the volar aspect of her little finger. This was sent for standard and mycobacterial culture and TB PCR (Hain Genotype MTBC Commercial Kit), which subsequently grew $M$ bovis thereby confirming the diagnosis of a TB tenosynovitis of the right wrist extending to involve the right little finger.

\section{TREATMENT}

She was started on standard quadruple therapy of rifampicin, isoniazid, pyrazinamide and ethambutol with pyridoxine. The pyrazinamide was stopped once $M$ bovis was isolated due to intrinsic $M$ bovis resistance to this drug. Ethambutol was

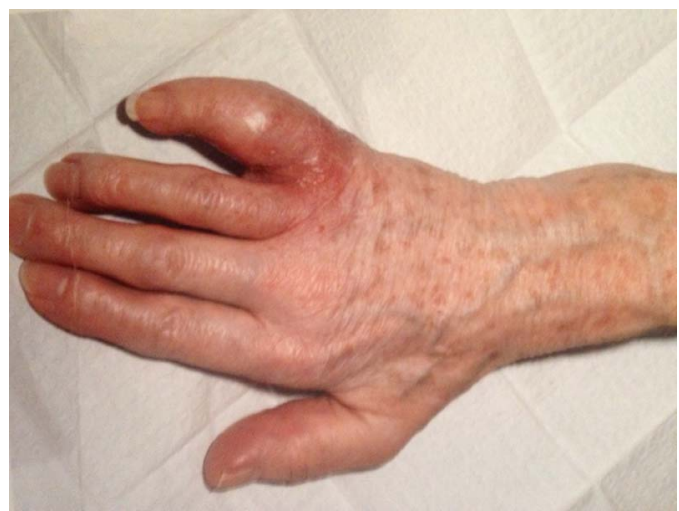

Figure 1 Tenosynovitis: dorsal aspect.

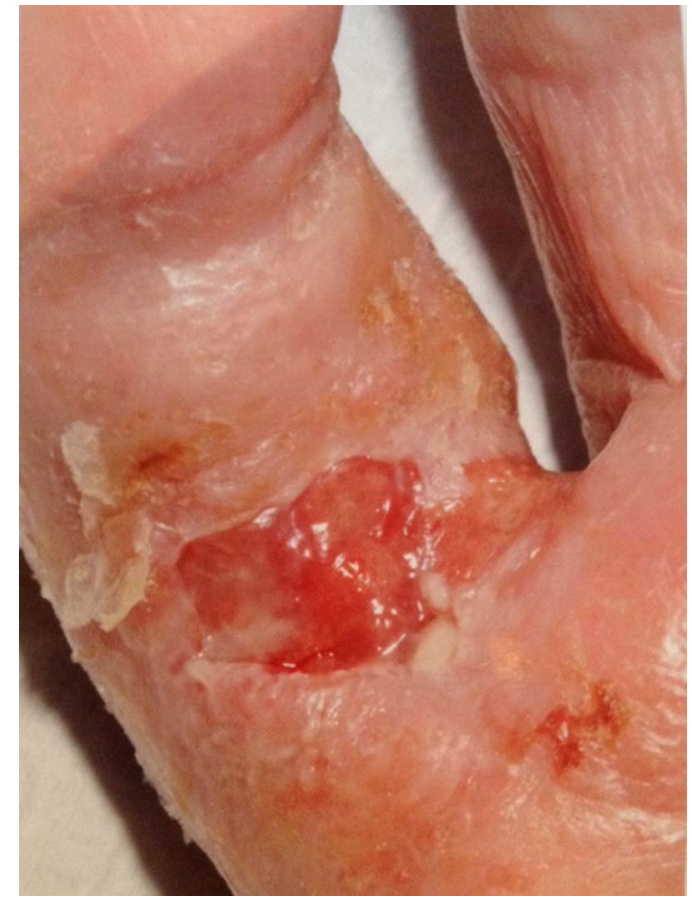

Figure 2 Tenosynovitis: palmar aspect.

stopped after 2 months of therapy and she continued on rifampicin and isoniazid therapy for a further 6 months $(9$ months total treatment); she tolerated the medication well with no significant side effects or complications with the treatment.

\section{OUTCOME AND FOLLOW-UP}

She is recovering well clinically, although there is a persistent fixed flexion deformity of her fifth metacarpal phalangeal joint for which she receives physiotherapy input. Her weight has also improved.

\section{DISCUSSION}

$M$ bovis is a member of a group of organisms with the capacity to cause tuberculosis in humans and is referred to as the Mycobacterium tuberculosis complex; other members include Mycobacterium africanum, Mycobacterium microti and Mycobacterium canetti. $M$ bovis is an aerobic, Gram-positive, rodshaped, acid-fast organism with a slow generation time of 16$20 \mathrm{~h}$. It is the primary cause of TB in cattle (Bovine TB); however, it is also recognised to be able to jump the species barrier and infect other animals including deer, badgers and many other mammals and may also infect humans. Human infection generally occurs in the setting of consumption of infected unpasteurised dairy products but may also occur through contact with infected animals or other humans via inhalation or contact with broken skin. ${ }^{1}$

$M$ bovis infection in humans may manifest with primary and postprimary forms and involvement may be pulmonary, extrapulmonary or disseminated. Infection via the aerosol route is most likely to result in pulmonary disease, whereas if it is acquired via ingestion of dairy products, then extrapulmonary disease is more likely, potentially affecting the lymph nodes, gastrointestinal tract, central nervous system, genitourinary system, skin and joints.

$M$ bovis as a cause of infectious tenosynovitis is extremely rare, only two cases being previously reported. Infectious tenosynovitis may develop via three main mechanisms: direct 
inoculation (bites, trauma), contiguous spread from infected adjacent structures and haematogenous spread. In the two previously published case reports direct inoculation was the identified mechanism with the patients occupation/past-time also being implicated: one case being that of a veterinarian whom accidentally inoculated himself with $M$ bovis from an infected possum ${ }^{2}$ and the second was that of a hunter who after shooting a whitetailed deer in Michigan, USA accidentally punctured his index finger with his hunting knife while field dressing the animal, thereby infecting himself. ${ }^{3}$ In the current case, although haematogenous spread from latent infection acquired by drinking unpasteurised milk as a child remains a possibility in the absence of any other risk factors identified from the history and examination, this is only hypothetical and the exact mechanism of infection remains uncertain.

The clinical features of tenosynovitis are a result of inflammatory or purulent fluid filling the synovial space between the tendons, thereby causing increased pressure within the synovial space resulting in pain (particularly on passive movements through the tendon) and swelling. The increasing pressure also acts to inhibit the inflammatory response and blood flow, thereby resulting in tendon ischaemia and increasing the possibility of complications to occur such as tendon necrosis and rupture. Inflammation of the tendon may also cause finger triggering and deformity as the thickened tendon becomes impinged on adjacent structures. Late signs may include cutaneous signs of ischaemia including necrosis. If infectious tenosynovitis is left untreated, the long-term complications may include tendon osteomyelitis, tendon destruction and rupture, deformity and profound functional impairment.

Differentiating between an infectious and non-infectious cause of tenosynovitis on clinical grounds is difficult with one study suggesting only $17 \%$ of patients with infectious tenosynovitis experienced fever. ${ }^{4}$ History and examination is important to identify any risk factors for an infectious aetiology, such as puncture marks, bites or trauma to the affected area. Occupation, foreign travel and pastimes are also of relevance. Imaging with ultrasound or MRI may be useful to evaluate the extent of inflammation/infection prior to surgery and the degree of tendon sheath abnormalities. Blood cultures should also be undertaken if signs of systemic infection are present. The diagnosis of infectious tenosynovitis, however, is usually confirmed by microbiological and histopathological investigations. Any suggestion of subcutaneous involvement (erythema, swelling, discharge) associated with the tenosynovitis should prompt specimens being taken and sent for Gram stain and culture. In the majority of cases of infectious tenosynovitis biopsy will be required for diagnosis and samples should be sent for both histopathological examination and culture for bacteria, fungal and mycobacterial organisms. The presence of granulomas on histopathological examination should raise the suspicion of mycobacterial infection and the appropriate histological stains should be undertaken.

Infection with $M$ bovis is indistinguishable both clinically and radiologically from $M$ tuberculosis infection. The members of the Mycobacterium complex are very similar; in fact, the attenuated bacillus Calmette-Guerin vaccine strain used to protect against $M$ tuberculosis infection is derived from a virulent strain of $M$ bovis. Initial investigations for mycobacteria such as staining for acid-fast bacilli and TB culture do not differentiate between the different mycobacterial species. A mono-resistance to pyrazinamide suggests $M$ bovis as the infective agent due to it being genotypically resistant.

Treatment of infectious tenosynovitis is usually with a combination of surgery and antibiotic therapy. In less advanced infection, in which the tendon sheath is viable, tendon sheath irrigation and drainage should be undertaken. In more advanced disease, surgical debridement of the tendon sheath and surrounding necrotic tissue is indicated. Amputation may be required in severe cases. Antibiotic treatment for $M$ bovis infection is similar to that of $M$ tuberculosis infection with the exception that $M$ bovis is intrinsically resistant to pyrazinamide. Therefore, empiric treatment should consist of rifampicin, isoniazid and ethambutol for 2 months and rifampicin and isoniazid for a further 7 months, depending on subsequent sensitivity testing, that is, a 9-month course rather than the standard 6 months. $M$ bovis, like $M$ tuberculosis, also has the capacity to acquire drug resistance; therefore, ensuring that treatment is adhered to and completed is essential.

\section{Learning points}

- Differentiating infectious and non-infectious tenosynovitis clinically is difficult and biopsy is often required to determine aetiology.

- The finding of granulomas on a synovial biopsy should always raise the suspicion of mycobacterial infection and should be investigated accordingly.

- Mycobacterium bovis is a rare but recognised cause of tenosynovitis and should be suspected if Mycobacterium species have been isolated and found to have pyrazinamide mono-resistance.

- Treatment of $M$ bovis tenosynovitis is with a combination of surgery and antibiotic therapy. Antibiotic therapy should consist of a prolonged course (9 months) of rifampicin, isoniazid and ethambutol, and this should be adhered to owing to the risk of developing antibiotic resistance.

Contributors JDU wrote the manuscript, organised digitisation of images and completed the consent process; $A B$ organised photographs to be taken and critically analysed the manuscript.

Competing interests None.

Patient consent Obtained.

Provenance and peer review Not commissioned; externally peer reviewed.

\section{REFERENCES}

1 Grange JM, Yates MD, De Kantor IN. Guidelines for speciation within the Mycobacterium tuberculosis complex. 2nd edn. World Health Organisation, 1996.

2 Cooke MM, Gear AJ, Naidoo A, et al. Accidental Mycobacterium bovis infection in a veterinarian. NZ Vet J 2002;50:36-8.

3 Wilkins MJ, Meyerson J, Bartlett PC, et al. Human Mycobacterium bovis infection and bovine tuberculosis outbreak, Michigan, 1994-2007. Emerg Infect Dis [serial on the Internet] 2008. http://wwwnc.cdc.gov/eid/article/14/4/07-0408.htm

4 Pang HN, Teoh LC, Yam AK, et al. Factors affecting the prognosis of pyogenic flexor tenosynovitis. J Bone Joint Surg Am 2007;89:1742. 


\section{Rare disease}

Copyright 2013 BMJ Publishing Group. All rights reserved. For permission to reuse any of this content visit http://group.bmj.com/group/rights-licensing/permissions.

BMJ Case Report Fellows may re-use this article for personal use and teaching without any further permission.

Become a Fellow of BMJ Case Reports today and you can:

- Submit as many cases as you like

- Enjoy fast sympathetic peer review and rapid publication of accepted articles

- Access all the published articles

- Re-use any of the published material for personal use and teaching without further permission

For information on Institutional Fellowships contact consortiasales@bmjgroup.com

Visit casereports.bmj.com for more articles like this and to become a Fellow 